\title{
Systolic anterior motion after mitral valve repair: Myectomy as an alternative solution
}

\author{
Giuseppe Rescigno, MD, Marco L. S. Matteucci, MD, \\ Attilio lacovoni, MD, Carlo Banfi, MD, Francesco Seddio, MD, \\ Luca Lorini, MD, Barbara Giamundo, MD, and Paolo Ferrazzi, MD, \\ Bergamo, Italy
}

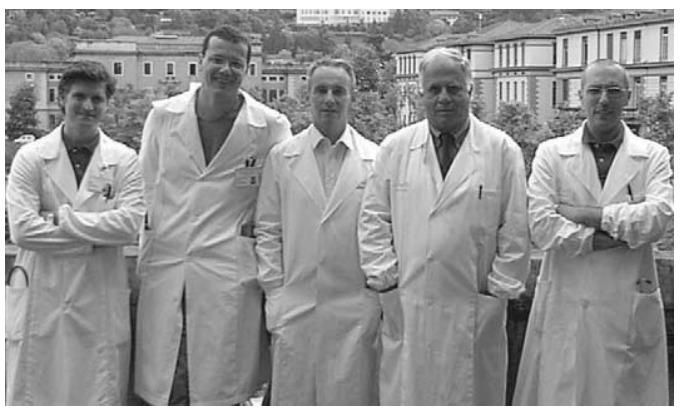

From left: Drs Seddio, Matteucci, Lorini, Ferrazzi, and Rescigno.
$\mathrm{M}$ itral repair represents the optimal choice to correct mitral valve insufficiency. Long-term results are significantly better with respect to mitral valve replacement. A rare complication of mitral valve repair is left ventricular outflow tract (LVOT) obstruction caused by systolic anterior motion (SAM) of the mitral apparatus. ${ }^{1}$

Correction of postrepair SAM is first medical: $\beta$-blockers and calcium-channel blockers might be of some help in reducing left ventricular contractility. However, when a severe LVOT obstruction develops, a more aggressive approach is generally needed. In some cases obstruction of the LVOT disappears after removing the prosthetic ring; in other cases mitral valve replacement is necessary. When a hypertrophic septum is suspected to be a contributing factor leading to obstruction of the LVOT, a possible solution might be to perform a septal myectomy. Here we describe 2 cases of postrepair LVOT obstruction that were successfully treated by means of a transaortic septal myectomy in patients with no preoperative obstruction of the LVOT.

\section{Clinical Summary}

Two female patients were referred to our center for severe mitral regurgitation. Patient 1 (67 years old) was in New York Heart Association Class (NYHA) III. Transesophageal echocardiography (TEE) showed a posterior leaflet prolapse with ruptured chordae and moderate anterior prolapse. Left ventricular function was normal (ejection fraction, 70\%), with moderate hypertrophy (diastolic septum thickness, $11.9 \mathrm{~mm}$ ). She was operated on for mitral valve repair. A posterior leaflet quadrangular resection was performed, which was associated with a posterior leaflet chordal transposition to the anterior leaflet free margin. A Duran 31-mm ring was implanted. During weaning from cardiopulmonary bypass (CPB), TEE showed a significant SAM with obstruction of the LVOT (peak gradient, $75 \mathrm{~mm} \mathrm{Hg}$ ) that persisted despite aggressive

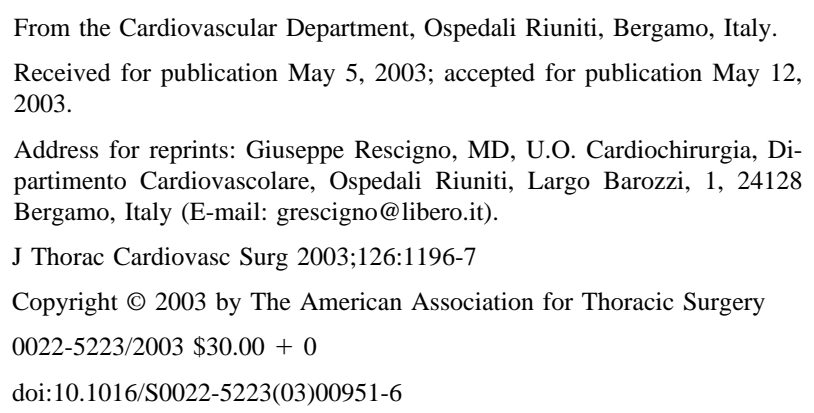

drug treatment (Figure 1). After a second cardioplegic arrest, the aorta was transversally opened, and a septal myectomy $(1.9 \mathrm{~g})$ was performed through the aortic valve. After aortic declamping, a new TEE control showed the complete regression of obstruction of the LVOT (Figure 2). The postoperative course was uneventful. This woman was discharged on the ninth postoperative day. Predischarge transthoracic echocardiography (TTE) revealed no mitral regurgitation and an absence of SAM. Pathologic examination revealed localized myocyte hypertrophy and interstitial fibrosis.

A follow-up echocardiogram performed 10 months after the operation showed a well-functioning mitral repair, with no residual regurgitation or stenosis. The LVOT had no gradient; septal thickness was $10 \mathrm{~mm}$. The patient is now in NYHA class II.

Patient 2 (69 years old) had severe dyspnea. Preoperative TEE showed a significant prolapse of both leaflets. Annular dilation was also present. The left ventricle had normal dimension and function. A high risk of SAM was reported by the cardiologist because of localized septal hypertrophy (diastolic thickness, $13 \mathrm{~mm}$ ) associated with abundant mitral valve tissue. At the time of the operation, a chordal transposition from the posterior to the anterior leaflet was performed, coupled with a quadrangular resection of the posterior leaflet without sliding because the remnant of the posterior leaflet was not redundant. No prosthetic ring was implanted to reduce the risk of obstruction of the LVOT. After discontinuation of $\mathrm{CPB}$, intraoperative TEE revealed a significant SAM (peak gradient, $60 \mathrm{~mm} \mathrm{Hg}$ ). CPB and cardioplegic arrest were reinstituted. Through an aortotomy, the patient underwent left ventricular septal myectomy. After declamping, TEE demonstrated a good result of valve repair without any residual LVOT obstruction. Microscopic examination of septal muscle showed mild myocyte hypertrophy. The postoperative course was uneventful. At follow-up (9 months), the patient was in NYHA class I; TTE demonstrated a well-functioning valve, with no residual regurgitation.

\section{Discussion}

We describe 2 cases of SAM with significant obstruction of the LVOT occurring after mitral valve repair, which were successfully treated by means of transaortic septal myectomy. A similar approach has been already described by Perier and coworkers. ${ }^{2}$ They reported a case of obstruction of the LVOT subsequent to mitral repair that was corrected by septal myectomy. However, in that patient a preoperative LVOT obstruction was already present, even if it was not considered significant. One should hypothesize that both diseases were present or even that mitral regurgitation was related to this forme fruste of hypertrophic obstructive cardiomyopathy. 


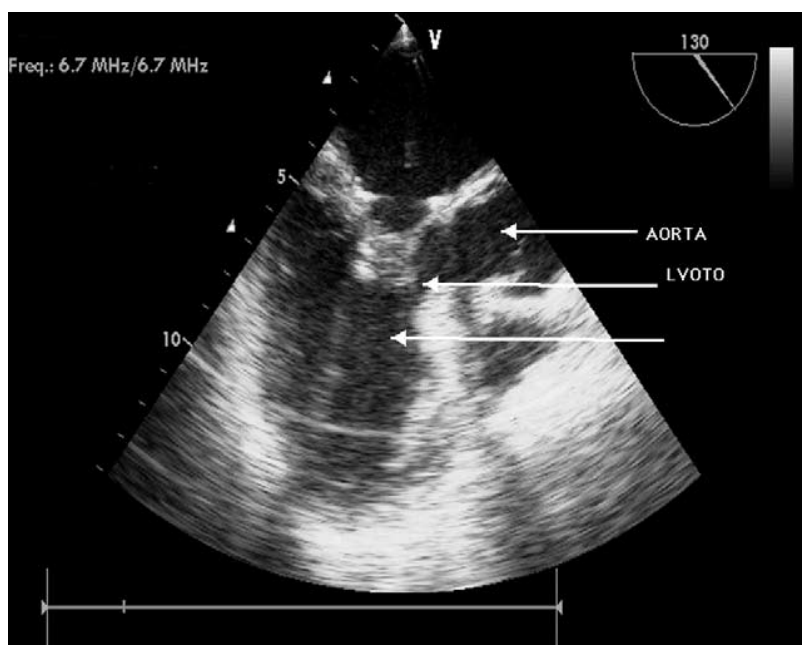

Figure 1. Echo frame showing the SAM after mitral valve correction. LVOT is significantly narrowed.

Dynamic obstruction of the LVOT caused by SAM is a wellknown complication after mitral valve repair. The incidence ranges from $2 \%$ to $16 \%$. $^{3}$ Several mechanisms have been documented; the most important seem to be the ratio between the length of the anterior and posterior leaflets, the length of the posterior leaflet, the distance between the coaptation rim and the ventricular septum, a small inner diameter of the left ventricle, the anterior displacement of the papillary muscles, and leaflet redundancy. ${ }^{4}$ All these factors could anteriorly displace the leaflet coaptation, and this condition is worsened by an associated septal hypertrophy. If these predisposing factors are preoperatively shown, surgical decision making should be modified accordingly. The sliding plasty of the posterior leaflet and flexible ring implantation are generally indicated. ${ }^{4}$ Nevertheless, none of these proposed techniques completely protect from the risk of SAM, the pathophysiology of which is still incompletely elucidated.

Our 2 patients presented a preoperative normal LVOT without gradient. In patient 2 the abundance of mitral valve tissue and a moderate left ventricular septal hypertrophy were only considered as predisposing factors to SAM. This was the reason for avoiding prosthetic ring implantation. Nonetheless, SAM developed. In fact, postrepair SAM and subsequent obstruction of the LVOT should be considered as a direct consequence of the repair itself.

When a significant LVOT obstruction develops after mitral repair operations, several options have been suggested. A pharmacologic approach might first be attempted, such as use of negative inotropes because $\beta$-blocker or calcium-channel blockers might help in reducing the gradient. More aggressive solutions are prosthetic ring explantation or mitral valve replacement. ${ }^{4}$

In our patients we adopted a different approach. Because a preoperative mild-to-moderate increase of septal thickness was present, we decided to perform a septal myectomy. This allowed a rapid and effective solution of LVOT obstruction. The postoperative course was uneventful, and follow-up echocardiography controls demonstrate a long-lasting, excellent result.

From a technical standpoint, this solution is easy to perform, requires a short additional crossclamping time, and does not re-

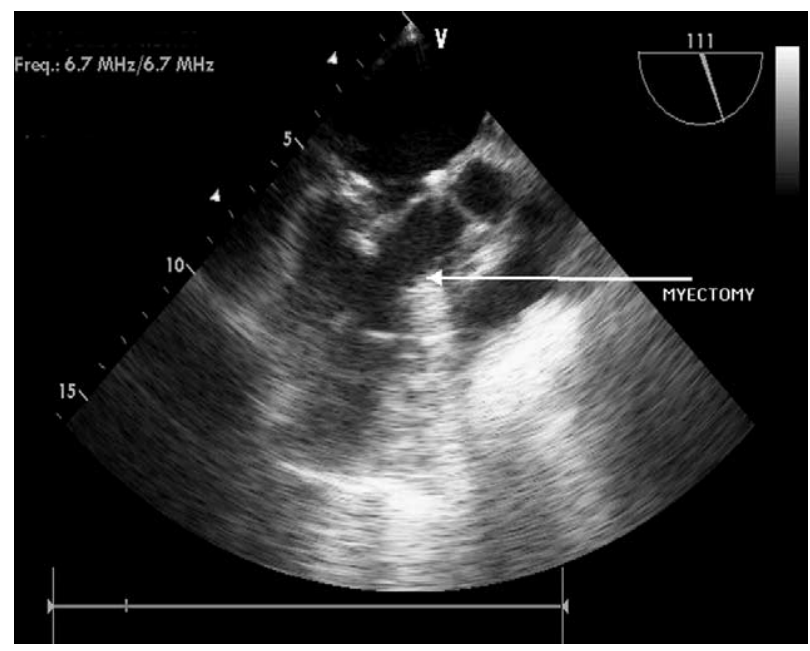

Figure 2. Echo frame captured after septal myectomy. SAM has been corrected.

quire a modification of the prior valve repair but is effectuated at another site in the cardiac chambers.

Some authors affirm that anterior displacement of the coaptation rim is the main factor leading to SAM and LVOT obstruction after mitral valve repair. Septal hypertrophy alone could not produce significant LVOT obstruction, despite flow velocities of 3.3 $\mathrm{m} / \mathrm{s}^{5}$ However, we believe that septal thickness contributes to reduction of the distance between the coaptation rim and the ventricular septum, which is a well-defined risk factor of LVOT obstruction. Septal trimming might reduce the Venturi effect, thus avoiding the suction effect on the anterior leaflet.

We can conclude that when a postrepair LVOT obstruction is intraoperatively documented and not corrected by means of pharmacologic intervention, a septal myectomy might be proposed if significant septal hypertrophy is demonstrated. It must be stressed that septal myectomy should be wisely performed. In fact, because septal thickness is often only moderately increased, the risk of iatrogenic ventricular septal defect is relatively high, and some experience with this kind of operation is mandatory.

\section{References}

1. Jebara VA, Mihaileanu S, Acar C, Brizard C, Grare P, Latremouille C, et al. Left ventricular outflow tract obstruction after mitral valve repair. Results of the sliding leaflet technique. Circulation. 1993;88:30-4.

2. Perier P, Hagen T, Stumpf J. Septal myectomy for left ventricular outflow tract obstruction after mitral valve repair. Ann Thorac Surg. 1994;57:1328-30

3. Maslow AD, Regan MM, Haering JM, Johnson RG, Levine RA. Echocardiographic predictors of left ventricular outflow tract obstruction and systolic anterior motion of the mitral valve after mitral valve reconstruction for myxomatous valve disease. J Am Coll Cardiol. 1999;34: 2096-104.

4. Lee KS, Stewart WJ, Lever HM, Underwood PL, Cosgrove DM. Mechanism of outflow tract obstruction causing failed mitral valve repair-anterior displacement of leaflet coaptation. Circulation. 1993; 88:24-9.

5. Lefebvre XP, He S, Levine RA, Yoganathan AP. Systolic anterior motion of the mitral valve in hypertrophic cardiomyopathy: an in vitro pulsatile flow study. J Heart Valve Dis. 1995;4:422-38. 